\title{
VELOCITY MEASUREMENT ON TWO-PHASE AIR BUBBLE COLUMN FLOW USING ARRAY ULTRASONIC VELOCITY PROFILER
}

\author{
MUNKHBAT BATSAIKHAN, ARI HAMDANI \& HIROSHIGE KIKURA \\ Department of Nuclear Engineering, Tokyo Institute of Technology, Japan.
}

\begin{abstract}
Two-phase flows can be encountered in a wide variety of industrial applications including a nuclear reactor, boilers, chemical reactor, etc. Bubble column is a simple and commonly used way to investigate two-phase flow due to their relatively simple construction and ease of operation. In the study of two-phase flow, the knowledge of gas and liquid velocities are required for better understanding of transport phenomena. Therefore, many measurement techniques have been developed such as LDV and PIV. However, those measurement techniques for velocity measurements require transparent test section and obviously fail if working fluid is opaque. To overcome this problem, there is another one technique, Ultrasonic Velocity Profiler (UVP), which can obtain the velocity information of both gas and liquid phases in non-transparent test section and opaque liquid. Originally, the UVP only measures one-dimensional velocity profile. For two-dimensional flow mapping, it is necessary to measure two velocity component at one spatial point to form a vector. In the present work, measurement system with two ultrasonic array sensors have been developed for two-dimensional velocity measurement and tested at two-phase bubbly column flow. The measurement results of UVP were compared with PIV measurement result to evaluate performance of the system. In two-phase bubbly flow, the performance of measurement system with ultrasonic array sensor is evaluated as 20\% comparing with PIV measurement results for one and two-dimensional velocity measurement.
\end{abstract}

Keywords: Two-phase bubble flow, ultrasonic array sensor, ultrasonic velocity profiler

\section{INTRODUCTION}

Two-phase flows can be encountered in a wide variety of industrial applications including nuclear reactors, boilers, chemical reactors, etc. It has been under a continuous investigation over the past several decades due to its complexity such as its turbulent phenomena, bubbles motion and so on. Bubble column is a commonly used way to investigate two-phase flow due to its relatively simple construction, ease of operation, and good mixing characteristics [1]. In the study of two-phase flows, the knowledge of velocity and volume fraction are very important for better understanding of transport phenomena in two-phase flow systems. Thus, many measurement techniques have been developed for volume fraction such as Electrical Resistivity Probe (ERP), Wire Mesh Sensor (WMS) and velocity such as Particle Image Velocimetry (PIV) and Laser Doppler Velocimetry (LDV), etc. A point measurement technique, such as ERP and LDV, can only measure the local volume fraction and velocity at a point. The WMS and PIV techniques can obtain the multi-dimensional information of velocity and volume fraction of both liquid and gas phases in two-phase flow. The PIV measurement technique applies to low volume fractions. As the number of bubbles increases, it becomes difficult to detect bubble sizes and positions. Moreover, this technique cannot be utilised with opaque liquids as it requires a transparent test section. To overcome these problems, there is another technique which is called ultrasonic velocity profiler (UVP).

The UVP method in single-phase flow was developed by Takeda [2]. The UVP can obtain the instantaneous velocity information in a transparent and opaque liquid such as liquid metal. The only requirement for this method to be effective is that a sufficiently high number 
of tracer particles is suspended in the fluid as an ultrasound reflector [2]. When UVP is applied to two-phase flows, ultrasonic pulses are reflected from both bubbles and tracer particles. Therefore, the data measured by the UVP monitor include the velocity information of both phases. Aritomi et al. [3] developed a system to measure the velocity profiles in bubbly flows using the UVP in countercurrent flow. It was difficult to use one transducer to measure both reflectors and bubbles because they require a different configuration of the transducer. Murakawa et al. [4] measured the velocity distribution using different diameters of the transducer and developed a multi-wave transducer. If a transducer diameter is selected properly, the velocity of each phase can be measured. The developed multi-wave transducer consists of two elements with different frequencies and diameters. The central element is used to measure the velocity of the liquid, and outer element is used for the velocity of bubbles. A separation technique based on the difference of intensities reflected on bubbles and particles was purposed. Echo signals of the ultrasonic beam reflected by bubbles are stronger than those reflected by tracer particles. The velocity measured by the multi-wave transducer in two-phase bubbly flow has validated by comparing with High-Speed Camera measurement by Kikura et al. [5].

In those previous studies, the UVP were used to investigate two-phase flow on one measurement line. To obtain information of multi-dimensional velocity using UVP, multiline measurement is required. Two- or three-dimensional velocity profile measurement for single phase flow by using UVP has been carried out by Kantoush et al. [6]. The application of multiple transducers has some drawbacks such as settling error and limitation of the installation position. To overcome them, an ultrasonic array sensor can be utilised as Kikura et al. [7] did in the air-water bubbly two-phase flow. An array sensor was used to measure the instantaneous and average velocity profile of single and two-phase flow by Kikura et al. [7]. It was a measurement of one-dimensional velocity. Hamdani et al. [8] developed a new approach to obtain two-dimensional velocity, and the approach was applied to single-phase and bubbly two-phase swirling flow. In the present work, measurement system with two ultrasonic array sensors have been developed for two-dimensional velocity measurement and tested at twophase bubbly column flow. A filtering technique, similar to the one proposed by Murakawa et al. [4] was utilised. The measurement results of UVP were compared with PIV measurement result to evaluate performance of the system.

\section{ULTRASONIC VELOCITY PROFILER}

The UVP method is based on pulsed ultrasound echography [9]. An ultrasound pulse is emitted from the transducer (TDX) along the measuring line, and the same transducer receives the echo reflected from the surface of tracer particles, bubbles, etc. The pulse reflection from these particles is recorded in an echo signal. The echo signal is Doppler-shifted according to reflector's velocity. Therefore, the velocity of the tracer particle can be obtained by analysing several successive reflections. During the measurement, a pulse is emitted in the interval, which corresponds to a pulse repetition frequency. Several echo sequences are needed to obtain a velocity profile, at least 2 and typically 128 sequences. The distance $x$ between each measurement volume and TDX can be calculated from the time delay $T$ and sound velocity $c$ as

$$
x=\frac{c T}{2} .
$$

Since the Doppler shift of the received echo signal is proportional to the velocity, the velocity is reconstructed as 


$$
\mathrm{u}=\frac{\mathrm{c} f_{d}}{2 f_{0}}
$$

where, $f_{0}$. is basic frequency of transducer. The signal proceing to detect the Doppler shift frequency $f_{d}$ is the main part of UVP method. To find out the Doppler shift frequency, many signal processing methods has been developed, e.g. Ref [10].

In velocity profile measurement of bubbly flow using UVP, it is important to know whether bubbles are crossing the measurement line or not. If a bubble crosses the measurement line, recorded amplitude by UVP will be higher compared to the amplitude when there is no bubble. Murakawa et al. [4] developed a separation technique to decide if there is a bubble on the measurement line using reflection intensity. In this work, a similar approach was used. First, the amplitudes were recorded in water without a bubble. An amplitude threshold is set as 2.5 times the average amplitude with no bubble. If amplitude is higher than the threshold, there is a bubble on the measurement line. On the other hand, if the amplitude is lower than the threshold, it is assumed that there is no bubble on the measurement line.

Originally, the UVP only measures one-dimensional velocity profile. For two-dimensional flow mapping, it is necessary to measure two velocity components at one spatial point to form a vector. Two velocity components are known at any intersection of measuring lines of any two transducers. An example of a two-dimensional flow mapping by two transducers is shown in Fig. 1. There, $\mathrm{u}_{1}$ and $\mathrm{u}_{2}$ are velocity components that are measured directly by individual transducers. Velocity along beam axis is expressed by eqns ( 3 and 4 ). Based on eqns ( 3 and 4), velocity vectors in Cartesian coordinates are reconstructed using eqns (5 and 6), respectively.

$$
\begin{aligned}
& u_{1}=\left(\begin{array}{l}
V \\
U
\end{array}\right)\left(\begin{array}{c}
\cos \theta_{1} \\
-\sin \theta_{1}
\end{array}\right) . \\
& u_{2}=\left(\begin{array}{l}
V \\
U
\end{array}\right)\left(\begin{array}{l}
-\cos \theta_{2} \\
-\sin \theta_{2}
\end{array}\right) .
\end{aligned}
$$

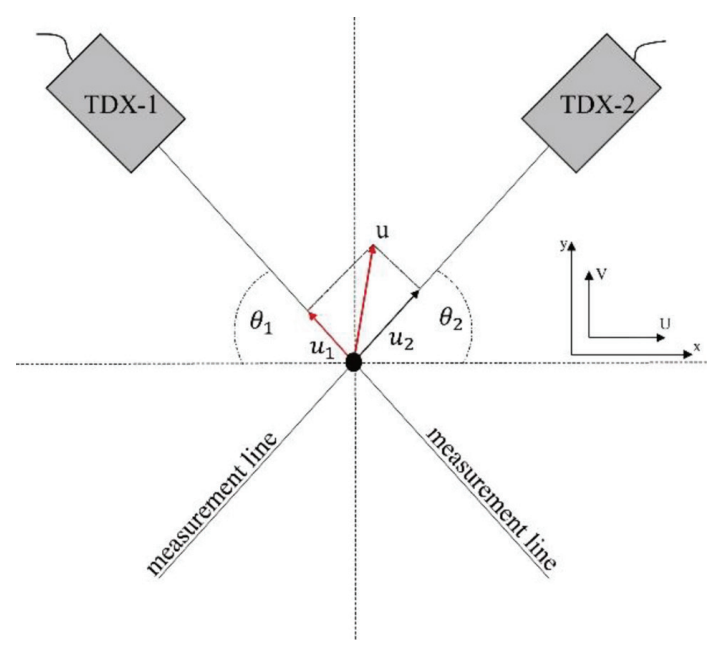

Figure 1: Vector decomposition based on two measurement lines. 


$$
\begin{gathered}
U=\frac{u_{2} \cdot \cos \theta_{1}+u_{2} \cdot \cos \theta_{1}}{-\sin \theta_{1} \cos \theta_{2}-\sin \theta_{2} \cos \theta_{1}} . \\
V=\frac{u_{1}+U \cdot \cos \theta_{1}}{\cos \theta_{1}}
\end{gathered}
$$

\section{EXPERIMENTAL APPARATUS AND PROCURES}

A simultaneous measurement carried out by UVP and High-Speed Camera (FastCam SA5 model 1300K-M3). The UVP equipment used in this experiment was UVP-DUO monitor with a multiplexer from Met-Flow SA, Switzerland. The experimental configuration is presented in Fig. 2. A rectangular acrylic box (300 mm wide, $300 \mathrm{~mm}$ deep and $300 \mathrm{~mm}$ high) was used due to good transparency for the measurement of HCS. Tap water was utilised as a liquid, and its level was the $280 \mathrm{~mm}$. Two sectorial array sensors were placed inside the box at the height of $200 \mathrm{~mm}$ from the bottom wall, the distance between two transducers was $118 \mathrm{~mm}$ (see Fig. 4). Both were controlled by UVP-DUO monitor. The HSC and light were placed perpendicular to the box to record image sequence of the bubble motion. The UVP monitor and HSC were synchronised. A porous material, $62 \mathrm{~mm}$ in length and $15 \mathrm{~mm}$ in diameter, was utilised as a gas distributor and placed at the centre of the box (see Fig. 4). The air was supplied by an air compressor, and the air flow rate through the gas distributor was measured by a rotameter (P200 Tokyo Keiso) and controlled by the control valve. Measurement range of the rotameter is from 0.06 to $0.6 \mathrm{~L} / \mathrm{min}$ and accuracy of the flowmeter is $\pm 3 \%$.

Two sectoral array sensors were used in the present experiment as shown in Fig. 3. The specification of array sensors is described in Table 1. Each of elements emits and receives the ultrasound individually. Figure 4 illustrates measurement sections of the UVP and PIV in detail. The red lines represent the measurement lines of the UVP. A dotted blue line represents the edge of the video taken by HSC. A ruler was attached on back side of the Sensor-2 for calibration of PIV. Flow condition of the entire measurement is listed in Table 2.

In UVP measurement, the array sensors were placed at some distance from the flow to minimise the disruption of the main bubbly flow, and 5000 instantaneous velocity profiles for each element were recorded at sampling rate $19 \mathrm{~ms}$. Measurement parameter of UVP are

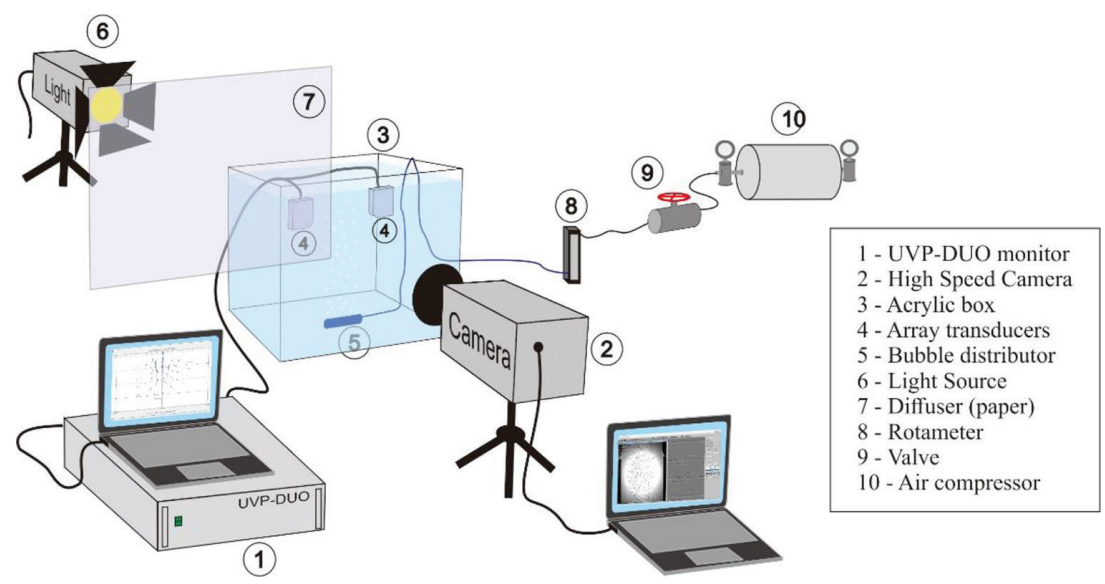

Figure 2: Experimental apparatus. 


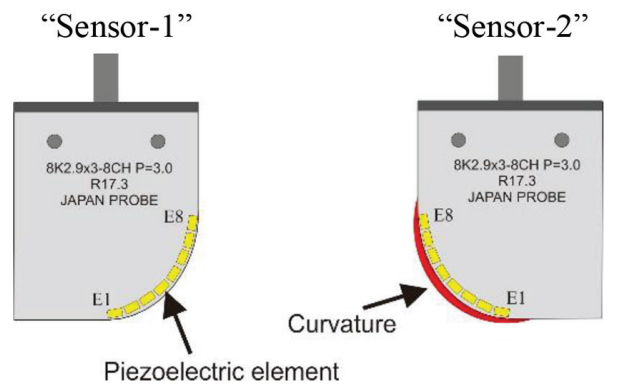

Figure 3: The arrangement of sectorial array sensors.

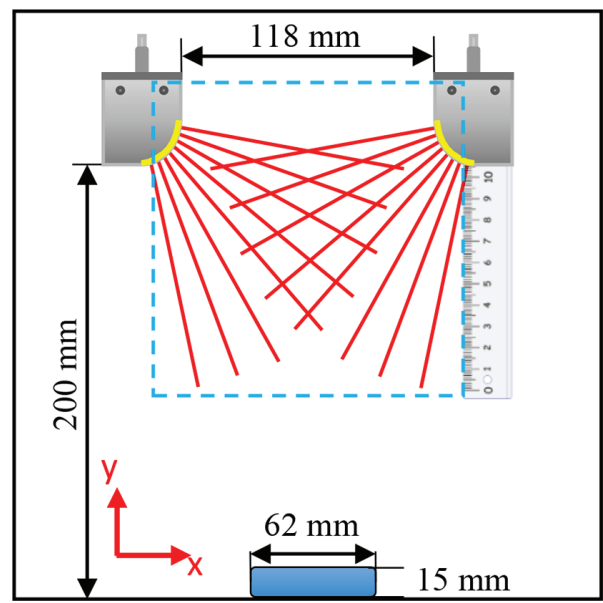

Figure 4: Test section.

Table 1: Specification of array sensors.

\begin{tabular}{ll}
\hline Basic frequency [MHz] & 8 \\
Number of elements & 8 \\
Wavelength [mm] & 0.19 \\
Element size [mm] & $2.9 \times 3$ \\
Pitch [mm] & 3 \\
Curvature [mm] & 17.3 \\
Height [mm] & 45 \\
Width [mm] & 35 \\
Thickness [mm] & 22 \\
\hline
\end{tabular}

show in Table 3. In the measurement of HSC, the HSC camera ( $896 \times 896$ pixel, $500 \mathrm{fps}$ ) providing a resolution of $0.16 \mathrm{~mm} / \mathrm{pixel}$ was used to record images of the flow. In this way, only $143.36 \mathrm{~cm}$ long part of the column was recorded for $20 \mathrm{~s}$ since it represents the most interesting part of the column. The PIV was performed with the use of MATLAB [12]. Image sequence of the bubble motion was recorded, and PIV processing was used to determine the average bubble velocity from these sequences. Illumination from the rear was used to obtain 
Table 2: Flow condition.

\begin{tabular}{ll} 
Working fluid: & \\
- Liquid & tap water \\
- Gas & air \\
System pressure & atmospheric \\
Water temperature $\left({ }^{\circ} \mathrm{C}\right)$ & $14-15$ \\
Bubble diameter $[\mathrm{mm}]$ & $0.1-2.3$ \\
Gas flow rate $[\mathrm{L} / \mathrm{min}]$ & 0.2 \\
\hline
\end{tabular}

Table 3: Parameters of UVP.

\begin{tabular}{ll}
\hline Channel distance [mm] & 0.73 \\
Channel width [mm] & 0.73 \\
Number of cycles & 8 \\
Number of repetitions & 64 \\
Length of measurement line $[\mathrm{mm}]$ & 105.5 \\
Sampling time $[\mathrm{ms}]$ & 19 \\
Sound velocity $[\mathrm{m} / \mathrm{s}]$ & 1465 \\
\hline
\end{tabular}

bubble shadows in a large area of the column. Binary images of bubbles were obtained by applying a threshold to the greyscale images and velocities were obtained from the subsequent images using PIV. Interrogation area of $60 \times 30$ pixels in the first pass, $30 \times 15$ pixels in the second pass and $16 \times 8$ pixels in the third pass were used with a 50\% overlap. Velocities were obtained by averaging 10,000 subsequent images of the bubble motion.

\section{RESULTS AND DISCUSSION}

\subsection{One-dimensional velocity profile by UVP and PIV}

One-dimensional velocity profile in vertical direction measured by UVP, assuming vertical direction of bubbly flow, was compared with PIV results. First three elements (from element No. 1 to element No. 3) of "Sensor 1" and "Sensor 2" were out from main flow. Therefore, other five elements at different angles (from element No. 4 to element No. 8) of "Sensor 1" and "Sensor 2" were used to measure one-dimensional velocity profiles, and ten velocity profiles were measured by UVP. In this section, two measurement results out of them, which are the best (Fig. 5) and worst agreement (Fig. 6) with PIV result, will be discussed. Figure 5 represents one-dimensional velocity profile measured by element 4 of 'Sensor 1' compared with the PIV results. As mentioned in the previous section, the array sensors were placed at some distance from the flow to minimise the disruption of the main bubble flow. The starting point of the velocity profile in the horizontal axis is different for each element due to the sectorial shape of array sensor and different angle of measurement lines. The PIV measurement also started at around $28 \mathrm{~mm}$ far from the array sensor. Therefore, the horizontal axis of Fig. 5 starts from around $28 \mathrm{~mm}$. In velocity profile measurement of bubbly flow using UVP, it is important to know whether bubbles are crossing the measurement line or not. If a bubble crosses the measurement line, recorded echo amplitude by UVP will be higher compared to the amplitude when there is no bubble. First, the amplitudes were recorded in a water without 


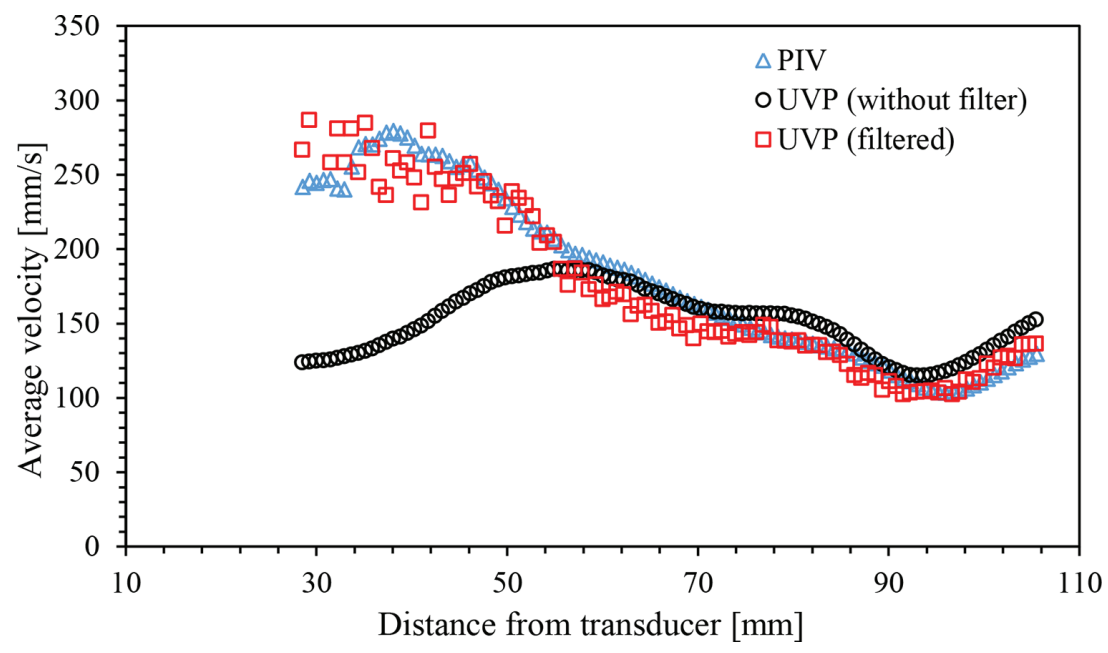

Figure 5: One-dimensional velocity profile by UVP (element No. 4 of 'Sensor-1') and PIV.

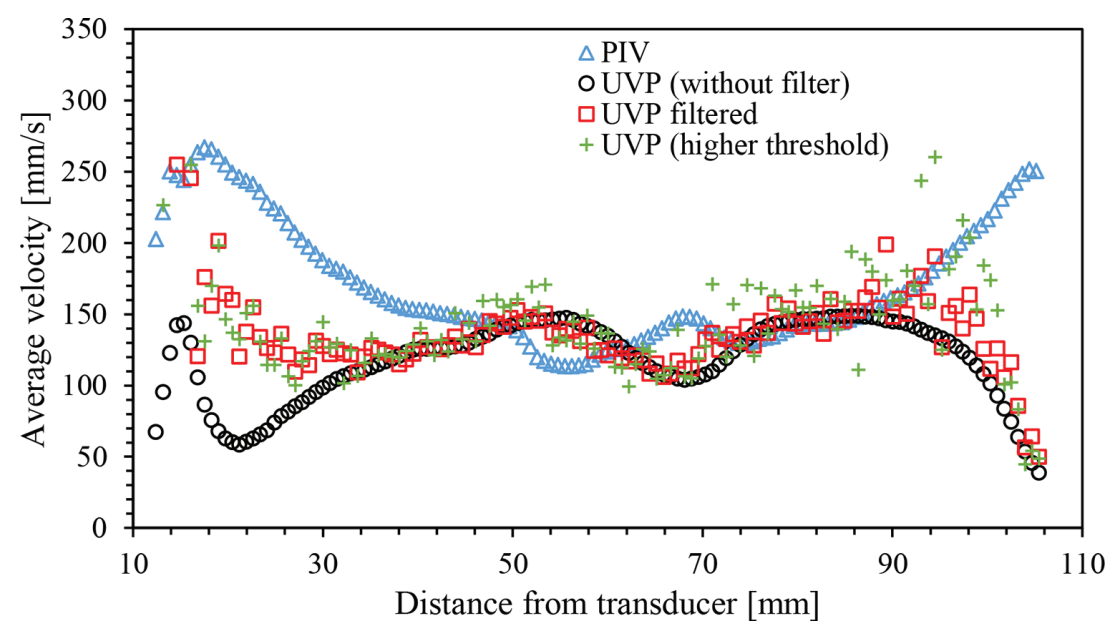

Figure 6: One-dimensional velocity profile by UVP (element No. 8 of 'Sensor-2') and PIV.

a bubble. An amplitude threshold is set as 2.5 times the average amplitude with no bubble. If amplitude is higher than the threshold, there is a bubble on the measurement line. On the other hand, if the amplitude is lower than the threshold, it is assumed that there is no bubble on the measurement line. As shown in Fig. 5, the comparison between results of PIV and UVP without filter has a large difference in the region from $28 \mathrm{~mm}$ to $50 \mathrm{~mm}$. After the measurement data were filtered out by amplitude, the UVP has a good agreement with PIV result. On the other hand, all the measurement results did not agree that well with PIV results despite being filtered by amplitude. Figure 6 shows the one-dimensional velocity profile measured by element No. 8 of 'Sensor 2' and compared with the PIV results. The filtering by amplitude is giving a little better result than without filter. Increasing threshold to 3.5 times higher than amplitude without bubble, improves the result, but velocity profile is becoming spread (see Fig. 6). Same phenomena were observed at element 8 of 'Sensor 1'. This difference will effect to 


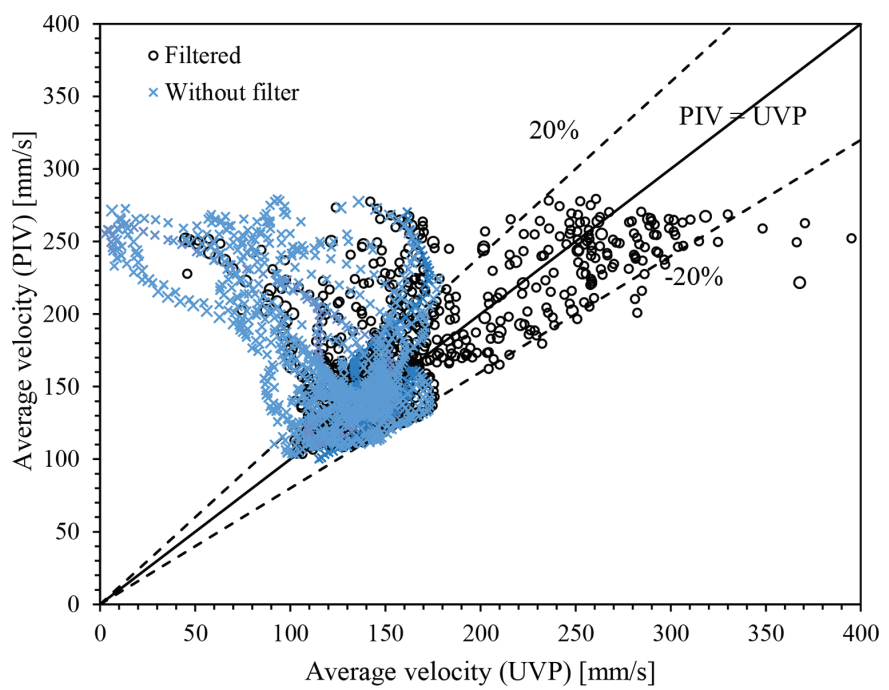

Figure 7: Difference between measured velocities by UVP (all 10 profiles) and PIV.

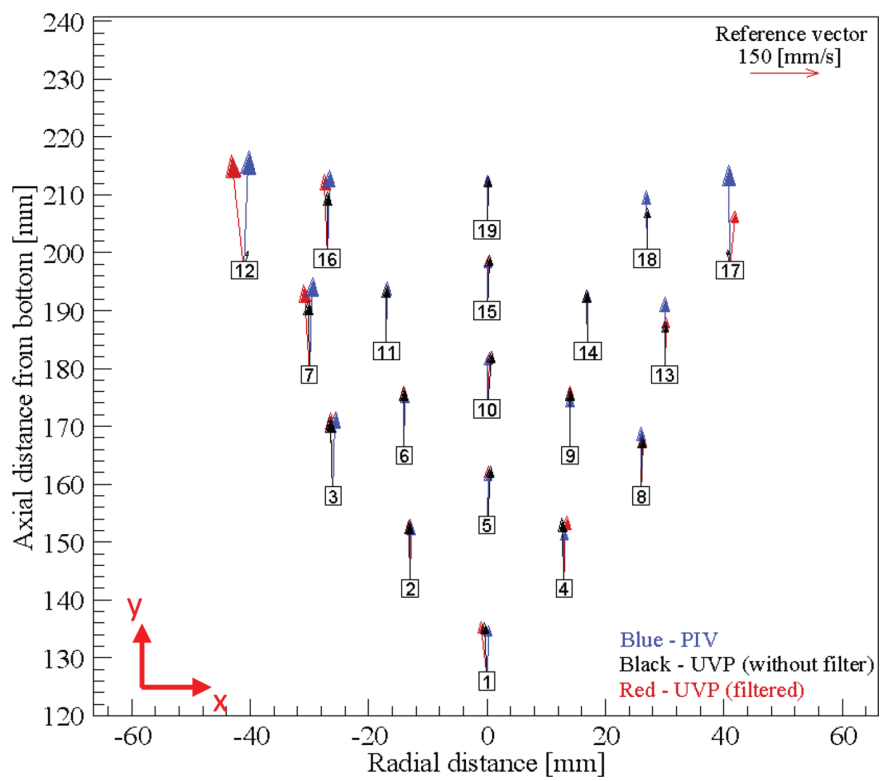

Figure 8: Results of flow mapping by UVP and PIV.

two-dimensional vector mapping where measurement lines of element 8 are crossing other measurement lines. The difference between one-dimensional velocities measured by UVP (all 10 profiles with and without filter) and PIV are shown in Fig. 7. 
Table 4: Quantitative comparison of velocities measured with the UVP and PIV.

\begin{tabular}{lcclccr}
\hline \multirow{2}{*}{$\begin{array}{l}\text { Measurement } \\
\text { points }\end{array}$} & \multicolumn{2}{l}{ UVP (without filter) } & \multicolumn{3}{c}{ PIV } & \multicolumn{2}{c}{ UVP (filtered) } \\
\cline { 2 - 6 } 1 & $\mathrm{~V}[\mathrm{~mm} / \mathrm{s}]$ & $\mathrm{U}[\mathrm{mm} / \mathrm{s}]$ & $\mathrm{V}[\mathrm{mm} / \mathrm{s}]$ & $\mathrm{U}[\mathrm{mm} / \mathrm{s}]$ & $\mathrm{V}[\mathrm{mm} / \mathrm{s}]$ & $\mathrm{U}[\mathrm{mm} / \mathrm{s}]$ \\
\hline 2 & 131.0 & -5.7 & 124.6 & 3.7 & 133.5 & -13.5 \\
3 & 151.8 & -1.7 & 147.1 & 2.4 & 153.3 & 0.0 \\
4 & 175.7 & -6.5 & 188.2 & 5.5 & 184.9 & -5.7 \\
5 & 155.9 & -4.3 & 134.9 & 0.6 & 162.3 & 5.5 \\
6 & 131.5 & 8.9 & 121.9 & 2.8 & 131.7 & 3.9 \\
7 & 151.6 & -1.3 & 144.4 & 2.0 & 153.8 & -0.2 \\
8 & 164.0 & -2.3 & 216.0 & 7.2 & 200.1 & -12.5 \\
9 & 132.7 & 3.1 & 153.0 & -0.2 & 134.8 & 4.4 \\
10 & 149.7 & -0.3 & 135.2 & 0.3 & 154.1 & 1.0 \\
11 & 129.2 & 11.1 & 120.9 & 2.2 & 125.9 & 6.6 \\
12 & 147.4 & 0.4 & 155.6 & 2.6 & 147.4 & 0.4 \\
13 & 41.3 & 8.3 & 266.0 & 10.4 & 257.4 & -28.2 \\
14 & 116.9 & 0.8 & 172.6 & 0.0 & 126.7 & 2.4 \\
15 & 135.6 & -2.9 & 133.4 & -0.2 & 136.2 & -1.3 \\
16 & 124.2 & 5.3 & 118.0 & 2.2 & 120.5 & 3.2 \\
17 & 148.4 & -0.2 & 198.0 & 5.0 & 188.9 & -5.6 \\
18 & 45.1 & -4.5 & 233.7 & -2.5 & 132.4 & 11.8 \\
19 & 113.1 & 0.4 & 150.1 & -1.0 & 113.1 & 0.6 \\
\hline & 118.0 & 2.7 & 120.7 & 1.9 & 118.3 & 1.1 \\
\hline
\end{tabular}

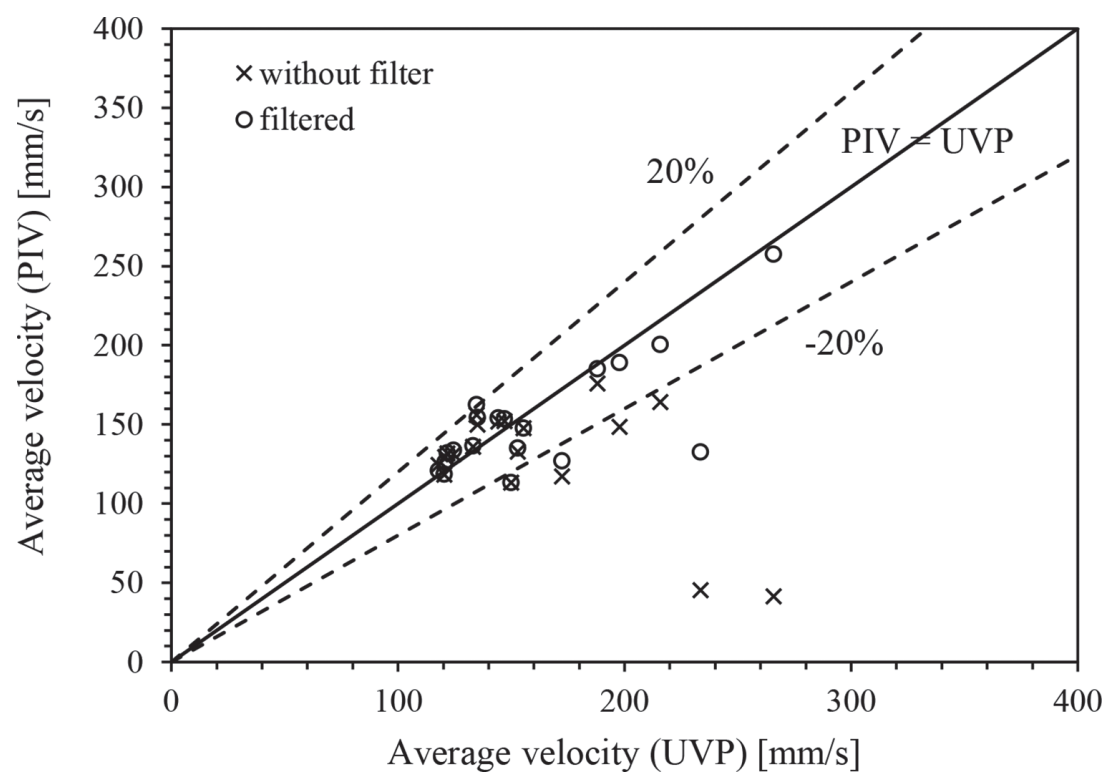

Figure 9: Comparison of velocity along the axial direction by UVP and PIV. 


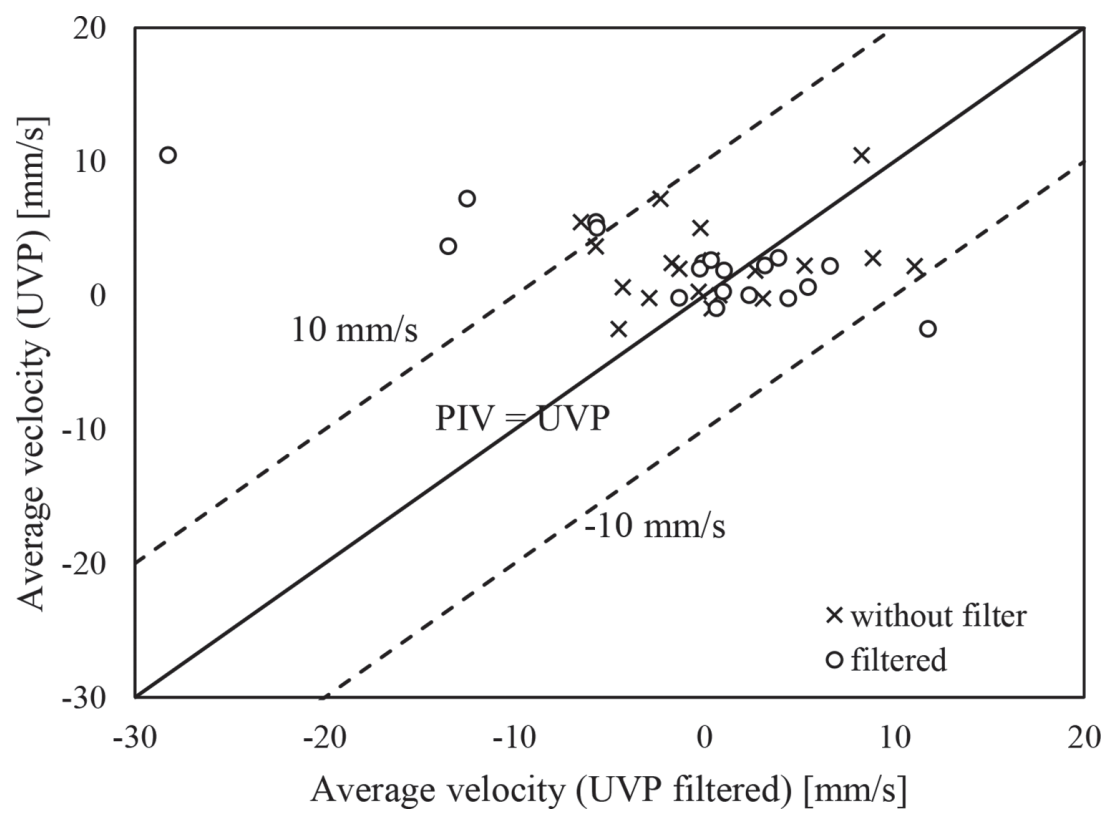

Figure 10: Comparison of velocity along radial direction by UVP and PIV.

\subsection{Flow mapping by UVP and PIV}

Figure 8 shows the two-dimensional flow mapping by UVP and PIV. To build velocity vector at spatial point in UVP, it is necessary to know the crossing point of two measurement lines. In this measurement, 19 points were crossed along the 10 measurement lines and two-dimensional vectors were extracted at those points. From PIV flow mapping results, velocity vectors are selected at same positions with UVP to compare. When using no filter, there are large differences at crossing points 12 (Element 5 of 'Sensor 1' and Element 8 of 'Sensor 2') and 17 (Element 8 of 'Sensor 1' and Element 5 of 'Sensor 2'), between UVP and PIV as shown in Fig. 8. That is because, as explained earlier, at the end of measurement lines of Element 8 for both sensors, there were large differences between results of PIV and UVP without filter. Table 4 shows a quantitative comparison between UVP and PIV. The difference of measured velocities along vertical and horizontal direction is showed in Figs 9 and 10, respectively.

\section{CONCLUSIONS}

Hamdani et al. [8] has developed two-dimensional UVP technique for the two-phase flow. However, the accuracy of the measurement system was not evaluated. In the present study, to evaluate the accuracy of the measurement system, two ultrasonic sectorial array sensors were applied to measure one- and two-dimensional velocity of bubbly two-phase flow in the rectangular bubble column. The measurement results of UVP were improved by using a filtering technique which is similar to the one proposed by Murakawa et al. [4] and compared with PIV result. The measurement results show that 
1. The filtering by amplitude is very important for accurate velocity profile measurement in two-phase flow.

2. After measured data had been filtered by amplitude, measurement results of UVP and PIV showed good agreement around $20 \%$.

3. The difference between two-dimensional flow measurement by UVP and PIV is estimated around $20 \%$ along the main flow (vertical) direction. Velocities difference in a horizontal direction is estimated around $\pm 10 \mathrm{~mm} / \mathrm{s}$.

Finally, the accuracy of the measurement system with ultrasonic array sensors for the twophase flow was evaluated as $20 \%$ for one- and two-dimensional velocity measurement.

\section{REFERENCES}

[1] Chen, R.C. \& Fan, L.S. Particle image velocimetry for characterizing the flow structure in three-dimensional gas-liquid-solid fluidized beds. Chemical Engineering Science, 47, pp. 3615-3622, 1992. https://doi.org/10.1016/0009-2509(92)85077-O

[2] Takeda, Y., Development of an ultrasound velocity profile monitor. Nuclear Engineering and Design, 126, pp. 277-284, 1991. https://doi.org/10.1016/0029-5493(91)90117-Z

[3] Aritomi, M., Zhou, S.,Nakajima, M., Takeda, Y., Mori, Y. \& Yoshioka, Y., Measurement system of bubbly flow using ultrasonic velocity profile monitor and video data processing unit. Journal of Nuclear Science and Technology, 33(12), pp. 915-923, 1996.

https://doi.org/10.1080/18811248.1996.9732033

[4] Murakawa, H., Kikura, H. \& Aritomi, M., Application of ultrasonic Doppler method for bubbly flow measurement using two ultrasonic frequencies. Experimental Thermal and Fluid Science, 29(7), pp. 843-850, 2005.

https://doi.org/10.1016/j.expthermflusci.2005.03.002

[5] Kikura, H., Murakawa, H. \& Aritomi, M., Velocity profile measurements in bubbly flow using multi-wave ultrasound technique. Chemical Engineering Communications, 172(2), pp. 114-133, 2009.

https://doi.org/10.1080/00986440902935705

[6] Kantoush, S.A., De.Cesare, G., Boillat, J.L. \& Schleiss, A.J., Flow field investigation in a rectangular shallow reservoir using UVP, LSPIV and numerical modelling. Flow Measurement and Instrumentation, 19, pp. 139-144, 2008.

https://doi.org/10.1016/j.flowmeasinst.2007.09.005

[7] Kikura, H., Hayashida, T., Ito, D., Aritomi, M. \& Mori, M., Application of linear ultrasonic array transducer to two-phase flow measurements. 6th International Symposium on Ultrasonic Doppler Methods for Fluid Mechanics and Fluid Engineering, pp. 95-98, 2008.

[8] Hamdani, A., Ihara, T. \& Kikura, H., Experimental and numerical visualizations of swirling flow in a vertical pipe. Journal of Visualization, 19, pp. 369-382, 2016. https://doi.org/10.1007/s12650-015-0340-8

[9] Takeda, Y., Velocity profile measurement by ultrasonic Doppler shift method. International Journal of Heat and Fluid Flow, 7, pp. 313-318, 1986.

https://doi.org/10.1016/0142-727X(86)90011-1 
[10] Kasai, C., Namewaka, K., Koyano, A. \& Omoto, R., Real-time Two-dimensional blood flow imaging using an autocorrelation technique. IEEE Transactions on Sonics and Ultrasonics, 32(3), pp. 458-464, 1985. https://doi.org/10.1109/T-SU.1985.31615

[11] "UVP Monitor - User's Guide" Model UVP-DUO with Software Version 3, Met-Flow.

[12] Thielicke, W. \& Stamhuis, E.J., PIVlab-Towards user-friendly, affordable and accurate digital particle image velocimetry in Matlab. Journal of Open Research Software, 2(1), e30, 2014.

https://doi.org/10.5334/jors.bl 